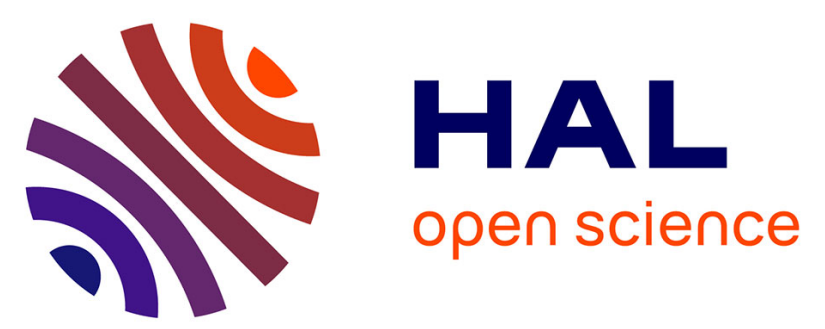

\title{
Caractérisation des interactions entre flux souterrains et superficiels dans un bassin andin en fonction de la variabilité spatio-temporelle du climat
}

Hervé Jourde, Mathieu Blanc, Romain Rochette, Denis Ruelland, Erick Hicks, Ricardo Oyarzun

\section{To cite this version:}

Hervé Jourde, Mathieu Blanc, Romain Rochette, Denis Ruelland, Erick Hicks, et al.. Caractérisation des interactions entre flux souterrains et superficiels dans un bassin andin en fonction de la variabilité spatio-temporelle du climat. La Houille Blanche - Revue internationale de l'eau, 2012, 2, pp.18 - 25. 10.1051/lhb/2012011 . hal-03202815

\section{HAL Id: hal-03202815 \\ https://hal.umontpellier.fr/hal-03202815}

Submitted on 20 Apr 2021

HAL is a multi-disciplinary open access archive for the deposit and dissemination of scientific research documents, whether they are published or not. The documents may come from teaching and research institutions in France or abroad, or from public or private research centers.
L'archive ouverte pluridisciplinaire HAL, est destinée au dépôt et à la diffusion de documents scientifiques de niveau recherche, publiés ou non, émanant des établissements d'enseignement et de recherche français ou étrangers, des laboratoires publics ou privés. 


\title{
Caractérisation des interactions entre flux souterrains et superficiels dans un bassin andin en fonction de la variabilité spatio-temporelle du climat
}

\author{
Hervé JOURDE ${ }^{1,3}$, Mathieu BLANC ${ }^{1,3}$, Romain ROCHETTE ${ }^{1,3}$, Denis RUELLAND ${ }^{2}$, \\ Erick HICKS ${ }^{3, *}$, Ricardo OYARZUN ${ }^{4,3}$
}

\author{
1. Université Montpellier 2 - UMR HydroSciences Montpellier, Place E. Bataillon, 34395 Montpellier Cedex 5, France - \\ e-mail : herve.jourde@um2.fr \\ 2. CNRS - UMR HydroSciences Montpellier, Place E. Bataillon, 34395 Montpellier Cedex 5, France \\ 3. CEAZA, Colina El Pino, Universidad La Serena, La Serena, Chile \\ 4. Departamiento Ingeniería de Minas Universidad La Serena, La Serena, Chile \\ *Actuellement à Minera Escondida, Antofagasta, Chile
}

\begin{abstract}
RÉSUMÉ. - Dans la région semi-aride du Norte Chico (Chili), la variabilité climatique induit une grande variabilité des ressources hydriques superficielles et souterraines. En prenant pour exemple le bassin amont de l'Elqui, nous montrons que, durant les évènements LNSO, les valeurs élevées du coefficient d'écoulement s'expliquent par une contribution des eaux souterraines aux écoulements de surface ; durant les années ENSO, les valeurs plus faibles du coefficient d'écoulement et la dynamique des variations piézométrique indiquent une recharge importante du compartiment souterrain. Sur la période 1977-2008 trois dynamiques d'interaction entre compartiments souterrains et superficiels sont identifiées durant les années hydrologiques caractérisées par un indice pluviométrique élevé : (i) l'augmentation logarithmique du niveau piézométrique, qui illustre une recharge rapide qui s'atténue ensuite, précède celle du débit du cours d'eau ; (ii) l'augmentation exponentielle du niveau piézométrique, qui montre une intensification progressive de la recharge en fonction du temps, succède à celle du débit ; (iii) l'augmentation du niveau piézométrique et du débit sont concomitants. Les dynamiques (i) et (ii) sont observées pendant des évènements ENSO, quand les précipitations sont distribuées sur de larges périodes ; les dynamiques (iii) sont observées pendant des années neutres, quand les précipitations de forte intensité surviennent sur une période de temps très courte. L'analyse de ces dynamiques et des coefficients d'écoulement montre que lorsque les précipitations moyennes annuelles sont inférieures à $70 \mathrm{~mm}$, l'équilibre relatif entre recharge du compartiment souterrain et ruissellement est rompu, ce qui peut engendrer de forts stress hydriques ; ce seuil pourrait être utilisé en tant qu'indicateur. Enfin, la variabilité spatiale des coefficients d'écoulement met en évidence une plus grande contribution du compartiment souterrain dans les bassins à forte composante granitique, ce qui semble indiquer une recharge en pied de versant depuis l'horizon altéritique développé dans les granites.
\end{abstract}

Mots clefs : variabilité hydro-climatique ; ressources en eau ; interactions surface/souterrain ; régime nival ; Río Elqui ; Chili.

\section{Characterization of the interactions between subterranean and superficial fluxes within an Andean catchment as a function of the spatio- temporal variability of climate}

\begin{abstract}
In the semi-arid region of Norte Chico (Chile), climate variability, mainly controlled by ENSO and LNSO events, generates a high variability of both surface water and groundwater fluxes. Taking the upper Elqui catchment as an example, this study found that, during LNSO events, the abnormally high values of the runoff coefficient may be the consequence of a groundwater contribution to surface water flow. During ENSO events, however, the lower values of the runoff coefficient and the dynamics of the water table level highlight the recharge of the subsurface compartment. For the hydrological years characterized by a high pluviometric index during the 1977-2008 period, three dynamics of interaction between groundwater and surface water are identified : (i) the water table increases before the river discharge, and its logarithmic increase highlights a rapid recharge related to the concomitance of snowmelt and rainfall events ; (ii) the water table increases after the river discharge and its exponential increase shows a progressive intensification of the recharge over time ; and (iii) the water table and the river discharge increase are concomitant. Dynamics (i) and (ii) are observed during the ENSO events, when precipitation occurs over a long time period ; dynamic (iii) is observed during the neutral years, when high intensity precipitations occur over short periods. The analysis of these dynamics and runoff coefficients shows that when the mean annual precipitation is less than $70 \mathrm{~mm}$, the relative equilibrium between runoff and recharge processes is broken, which may generate a high hydrological deficit ; this threshold could thus be used as a warning for drought. Finally, the spatial variability of runoff coefficients shows a larger contribution of the subsurface compartment in catchment with a larger proportion of granites, suggesting a mountain front recharge from the alterites in the granitic watershed..
\end{abstract}

Key words : hydro-climatic variability ; water resource ; surface/subsurface interactions ; snowmelt ; Río Elqui ; NorthCentral Chile. 


\section{INTRODUCTION}

Dans la région du Norte Chico au Chili, la variabilité naturelle du climat, contrôlée principalement par les phénomènes El Niño et La Niña, est à l'origine de grandes disparités dans l'intensité et la répartition des précipitations. Le bilan de masse est rarement respecté à l'échelle annuelle sur les bassins de cette région [Favier, 2009], ce qui s'explique en partie par le gradient orographique des précipitations et par la contribution des glaciers. Cependant, une sous-estimation de la contribution du compartiment souterrain pourrait avoir la même conséquence. Il apparaît donc primordial de mieux caractériser le fonctionnement des compartiments superficiels et souterrains de ces hydrosystèmes andins ainsi que leurs interactions. En prenant le bassin amont de l'Elqui comme exemple, les flux superficiels et souterrains sont analysés en fonction de la variabilité spatio-temporelle du climat entre 1977 et 2008 .

\section{MATERIELS ET METHODES}

Le bassin de l'Elqui (9 $700 \mathrm{~km}^{2}$, Fig. 1a), est l'un des trois principaux réseaux hydrographiques de la $\mathrm{IV}^{\mathrm{e}}$ région du Chili. Il est situé entre les latitudes $29^{\circ} 27^{\prime} \mathrm{S}$ et $30^{\circ} 34^{\prime} \mathrm{S}$ et les longitudes $71^{\circ} 22^{\prime} \mathrm{W}$ et $69^{\circ} 52^{\prime} \mathrm{W}$. Ce bassin s'étend de la côte pacifique à la ligne de crête de la cordillère des Andes qui dépasse localement 6000 mètres d'altitude. Comme la majorité des cours d'eau de la région, le río Elqui présente un débit avec une forte variabilité interannuelle $\left(2 \mathrm{~m}^{3} / \mathrm{s}\right.$ $<$ module annuel moyen $<33 \mathrm{~m}^{3} / \mathrm{s}$ ) et naît de la confluence des ríos Turbio et Claro (à $815 \mathrm{~m}$ d'altitude), ses deux principaux affluents, dont les bassins ont une superficie respective d'environ 4200 et $1550 \mathrm{~km}^{2}$. Le régime hydrologique de ces affluents est nival, typique de zones montagneuses arides, les débits les plus élevés étant enregistrés entre novembre et février.
D'un point de vue géologique, le bassin versant est constitué de quatre grands types de formation : les roches granitiques, les roches volcaniques, les dépôts volcanosédimentaires consolidés et les remplissages sédimentaires quaternaires. Les roches déposées durant le Mésozoïque et le Cénozoïque - essentiellement des roches ignées plutoniques (batholites) ou de caractère volcanique ou volcano clastique - présentent uniquement une perméabilité secondaire, produit de leur fracturation [Luengo, 2006]. Cette perméabilité est contrôlée par la direction actuelle des contraintes tectoniques, généralement différente de celle à l'origine de la fracturation. La fracturation (diaclases et joints tectoniques) joue un rôle important vis-à-vis de l'infiltration mais très limité vis-à-vis des circulations à grande échelle ; en revanche, malgré leur moindre abondance, les failles jouent un rôle majeur à la fois dans la circulation des eaux souterraines et dans les processus d'infiltration, notamment lorsqu'elles sont associées à une fracturation intense ou à des structures secondaires. C'est à la faveur de ces fractures et failles que se concentrent les écoulements vers des sources (Vertientes) localement utilisée pour l'alimentation en eau. Toutefois, aucune donnée de perméabilité concernant ces roches plutoniques et volcaniques n'est disponible, les forages étant positionnés essentiellement dans les remplissages sédimentaires quaternaires. Ces derniers, essentiellement d'origine alluviale et colluviale, peuvent atteindre une épaisseur de $200 \mathrm{~m}$ et représentent les principaux aquifères de la zone d'étude [USDI, 1959], susceptibles d'emmagasiner et de transmettre de l'eau en quantité importante [Miranda, 2003]. Dans les secteurs des ríos Turbio et Claro jusqu'à $3 \mathrm{~km}$ en aval de leur confluence (Algarrobal), les dépôts peuvent atteindre près de $150 \mathrm{~m}$ et leur perméabilité a été évaluée à $10 \mathrm{~m} / \mathrm{j}$, ce qui permet un transfert rapide d'importants volumes d'eau souterraine.

Dans cette région semi-aride, le climat se caractérise par des précipitations annuelles inférieures à $100 \mathrm{~mm}$ sur la côte et excédant $400 \mathrm{~mm}$ dans la cordillère où la hauteur de neige

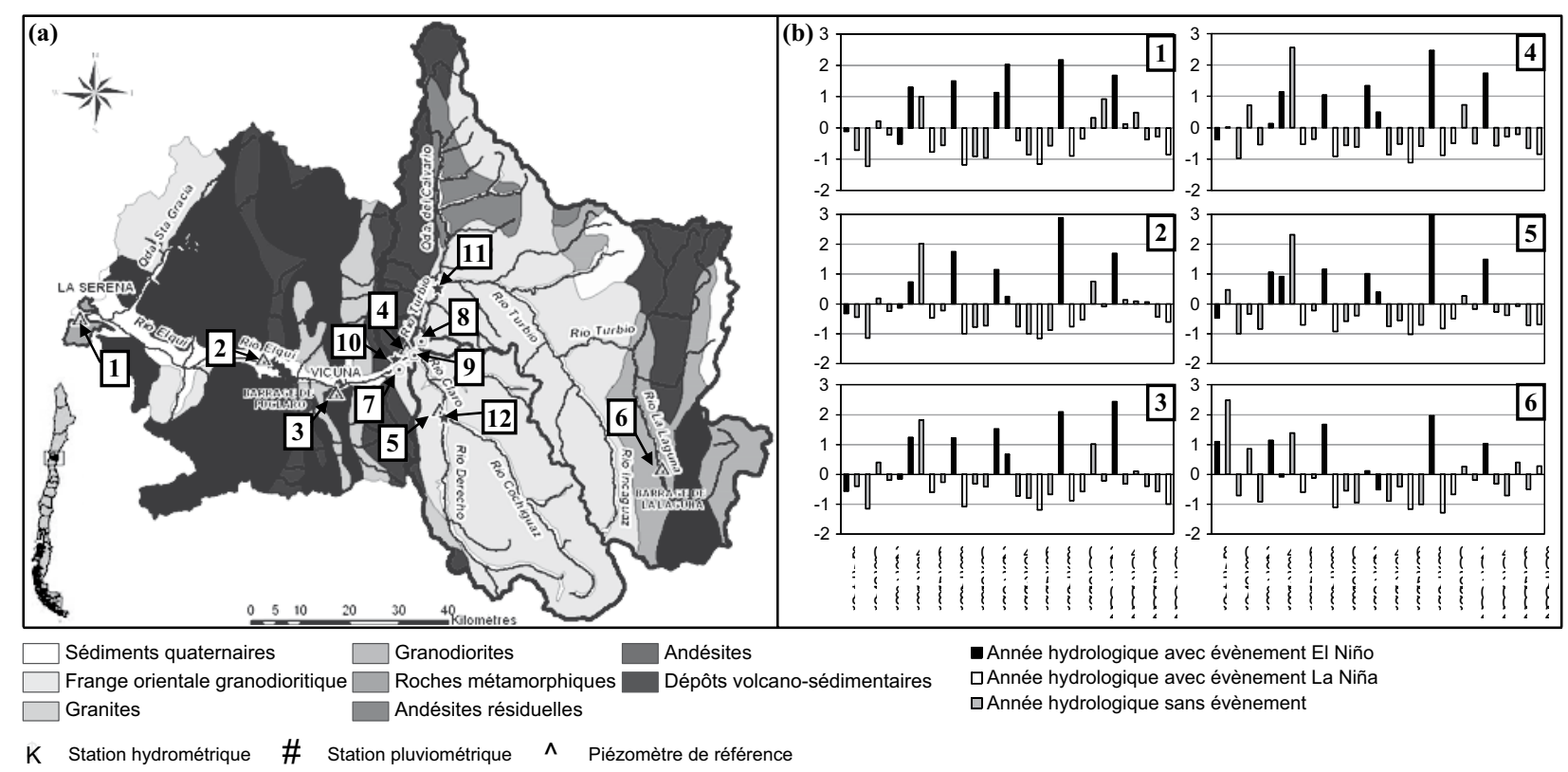

Fig. 1 (a) : Contexte général du bassin du río Elqui et réseau de mesures [d'après Cabezas, 2007] : stations pluviométriques (La Serena (1), Almendral (2), Vicuña (3), Rivadavia (4), Monte Grande (5) et La Laguna (6)), stations hydrométriques (río Elqui à Algarrobal (7), río Turbio à Varillar (8) et río Claro à Rivadavia (9)), et piézomètres de référence (Algarrobal (10), Bocalume (11), Monte Grande (12)) ; (b) Indices pluviométriques (IP) calculés pour la période 1977-2008 aux stations pluviométriques. 
peut atteindre plusieurs mètres durant des années exceptionnelles. Pour chaque année hydrologique (avril-mars), un indice pluviométrique $(I P)$ a été calculé :

$$
I P=\frac{x i-\bar{x}}{\sigma}
$$

où $x i$ indique la pluviométrie de l'année $\mathrm{i}, \bar{x}$ la pluviométrie moyenne interannuelle sur la période de référence et $\sigma$ l'écart type de la pluviométrie annuelle sur la période de référence.

Ces $I P$, qui traduisent un excédent ou un déficit pluviométrique par rapport à la période de référence choisie (valeurs centrées réduites), ont été confrontés aux données ONI (Oceanic Niño Index), basées sur des anomalies de température de la surface de l'océan Pacifique de la région NIÑO 3.4. Pour chacun des bassins (Fig. 1), un piézomètre d'observation représentatif de la dynamique naturelle du réservoir souterrain (puits sans pompage et/ou avec une grande variabilité interannuelle) ainsi qu'une station hydrométrique ont été considérées pour l'analyse des interactions entre compartiment souterrain et superficiel (Tableau 1) durant les années hydrologiques présentant un IP élevé. Quatre de ces années sont marquées par des événements El Niño : 1987-88, 1992-93, 1997-98 et 2002-03. Deux autres correspondent à des années neutres (sans évènements) : 1978-79 et 1984-85.

Des indices hydrologiques IH (valeurs centrées réduites de débit) et des indices piézométriques IPZ (valeurs centrées réduites de piézométrie) ont ensuite été calculés pour évaluer l'excès ou le déficit des flux superficiels et souterrains par rapport à la période de référence. Les piézomètres d'observation interceptent un complexe alluvionnaire multicouches, le premier horizon ( $0-40 \mathrm{~m}$ sous la surface) comprenant galets graviers et sables plus ou moins riches en argiles et limons, les horizons sous-jacents (jusqu'à $150 \mathrm{~m}$ sous la surface, localement d'avantage) correspondant à des niveaux de sables, graviers et limons plus ou moins argileux, intercalés avec des niveaux sablo-argileux ou sablo-limoneux. Concernant les différents ouvrages, le forage de Bocalume a une profondeur de $50 \mathrm{~m}$ tout comme celui de Monte Grande alors que l'ouvrage d'Algarrobal avait une profondeur de $130 \mathrm{~m}$ lors de la foration.

Afin de compléter l'analyse, des coefficients d'écoulement (CE) ont enfin été calculés :

$$
C E=\frac{E_{t}}{P},
$$

où $E_{t}$ désigne la quantité d'eau qui s'écoule chaque année à l'exutoire du bassin versant et $P$ la quantité annuelle d'eau précipitée sur le bassin versant calculée par une interpolation des précipitations selon la méthode des distances inverses pondérées [Ruelland, 2011] que Moralès et al. [2007] ont identifiée comme étant suffisamment précise, parmi d'autres méthodes de reconstruction de données. Les stations météorologiques sélectionnées sont celles offrant les chroniques observées présentant le moins de lacunes sur la période 1978-2008 de manière à capturer la variabilité hydroclimatique spatiale et temporelle du bassin. 15 stations pluviométriques ont ainsi été utilisées pour l'interpolation de champs de précipitations journaliers selon une grille régulière de $5 \mathrm{~km}$ de résolution spatiale. Bien que les précipitations varient sensiblement avec l'altitude, une spatialisation prenant en compte le gradient orographique Ouest-Est n'est pas apparue pertinente dans le cas du bassin de l'Elqui, également soumis à un gradient climatique Nord-Sud. Des validations croisées par comparaison à d'autres stations et une analyse de sensibilité d'un modèle hydrologique (Ruelland et al., 2011) à différents jeux de données de précipitations (prenant ou non en compte l'altitude) n'ont pas permis de mettre en évidence clairement la nécessité de considérer le gradient orographique pour l'interpolation des précipitations dans ce contexte. Néanmoins, les mesures utilisées provenant essentiellement des fonds de vallée, une incertitude sur l'estimation des précipitations existe et peut amener à nuancer certaines interprétations. De ce fait, si l'on considère une sous estimation probable de précipitations dans certains cas, alors, les coefficients d'écoulement peuvent se retrouver par conséquent légèrement surestimés.

Pour chaque bassin (Tableau 2), le terme $E_{t}$ tient compte des prélèvements par les canaux d'irrigation (données disponibles jusqu'à 1998-99). Le coefficient d'écoulement $(C E)$ est influencé par la géologie, la topographie, le type de sol, l'occupation du sol et le couvert végétal. Il permet d'avoir une idée de la capacité d'infiltration des sols et de l'évapotranspiration, mais peut aussi permettre d'identifier la contribution des écoulements souterrains aux écoulements de surface [Jourde, 2007].

\section{RÉSULTATS ET DISCUSSION}

\section{III.1. Analyse des indices hydroclimatiques}

Durant les années humides (IP élevés), l'analyse interannuelle des différents indices montre que les IH sont toujours supérieurs aux IPZ sur le bassin du río Turbio (Fig. 2), ce qui est simplement la conséquence d'une réponse aux précipitations plus rapide pour le compartiment superficiel que pour le compartiment souterrain. Durant l'année qui suit ces années humides, l'IPZ reste élevé et est dans la majorité des cas plus élevé que celui de l'année précédente, alors que l'IH diminue fortement. Ceci s'explique par la plus forte inertie du compartiment souterrain, caractérisée par une longue période de récession ( 1 à 2 ans) alors que la récession des débits s'opère sur des périodes plus courtes (4 à 6 mois). Durant les années qui suivent des années sèches au contraire, on observe une diminution des IH et IPZ du fait du déficit pluviométrique.

Tableau 1 : Altitude des piézomètres de référence et des stations hydrométriques pour les trois bassins.

\begin{tabular}{lll} 
& Piézomètres de référence & Station hydrométrique \\
\hline Bassin du río Turbio & Bocalume (altitude $1025 \mathrm{~m}$ ) & Varillar (altitude $860 \mathrm{~m}$ ) \\
Bassin du río Claro & Monte Grande (altitude $1111 \mathrm{~m}$ ) & Rivadavia (altitude $820 \mathrm{~m}$ ) \\
Bassin amont de l'Elqui & Algarrobal (altitude $767 \mathrm{~m}$ ) & Algarrobal (altitude $760 \mathrm{~m}$ ) \\
\hline
\end{tabular}


Tableau 2 : Coefficients d'écoulement (CE) sur les bassins des ríos Turbio et Claro et sur le bassin amont de l'Elqui. Les années hydrologiques en gris foncé et en gris clair correspondent respectivement à des évènements El Niño et La Niña. Les valeurs de CE grisées sont issues de séries de données présentant des lacunes

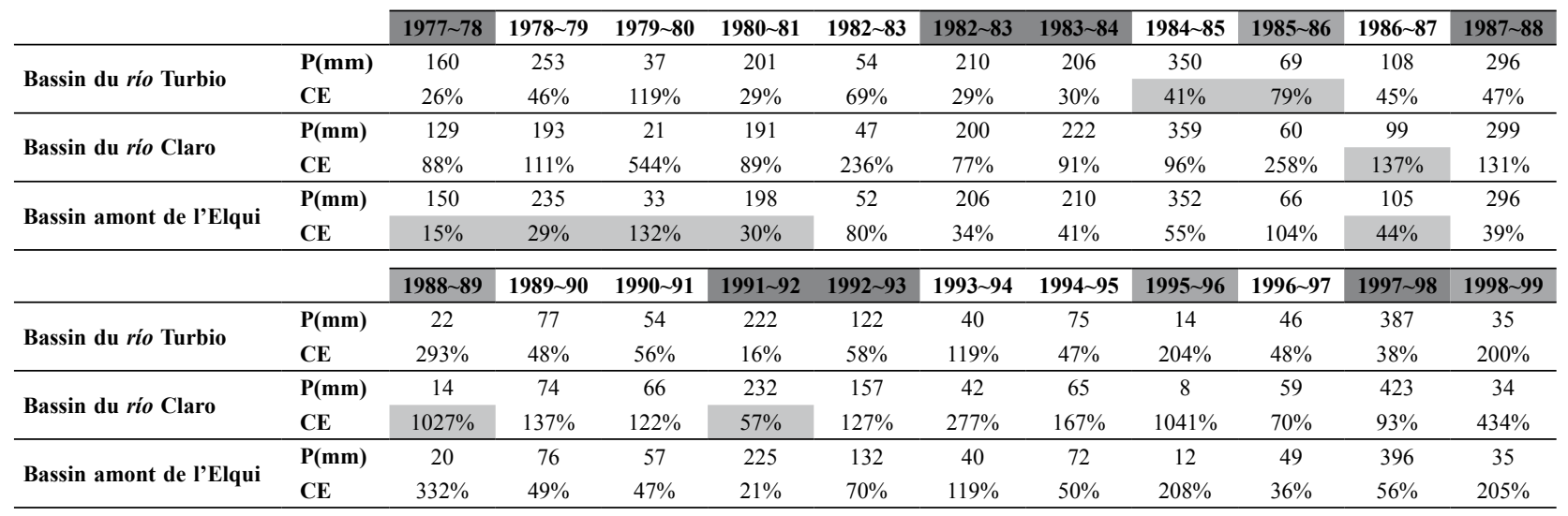

Sur le bassin du río Turbio, cette dynamique interannuelle est plus marquée que sur le bassin du río Claro ; comme le río Claro alimente des canaux et des réservoirs pour l'irrigation, une partie du ruissellement peut alors contribuer à la recharge de l'aquifère, ce qui augmenterait l'IPZ au détriment de l'IH. Ce comportement (Fig. 2) est observable durant des années sèches (IP faible) telles que 1986-87, 1994-95 et 1995-96, ce qui s'explique assez bien ; par contre, lorsqu'il se produit durant certaines années humides (IP élevé) telles que 1978-79 et 1992-93, l'irrigation ne peut plus l'expliquer.
Dans ce cas, la recharge du compartiment souterrain (IPZ > IH) pourrait être expliquée par la géologie puisque le substratum granitique fracturé, qui constitue plus de $80 \%$ de la superficie de ce bassin versant, pourrait contribuer notablement à la recharge du compartiment souterrain.

Sur le bassin amont de l'Elqui, les indices IPZ et IH ont un comportement globalement semblable à celui observé sur le bassin du río Turbio, ce qui souligne l'importance de cet affluent majeur dans la dynamique générale des interactions entre compartiments superficiels et souterrains.

(a)

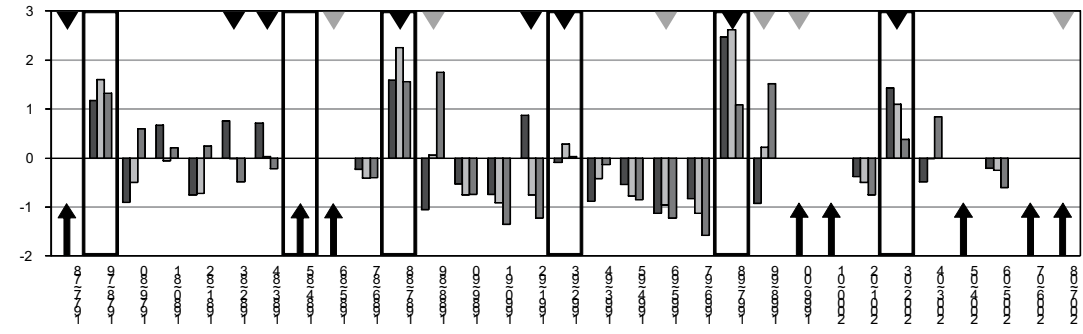

(b)

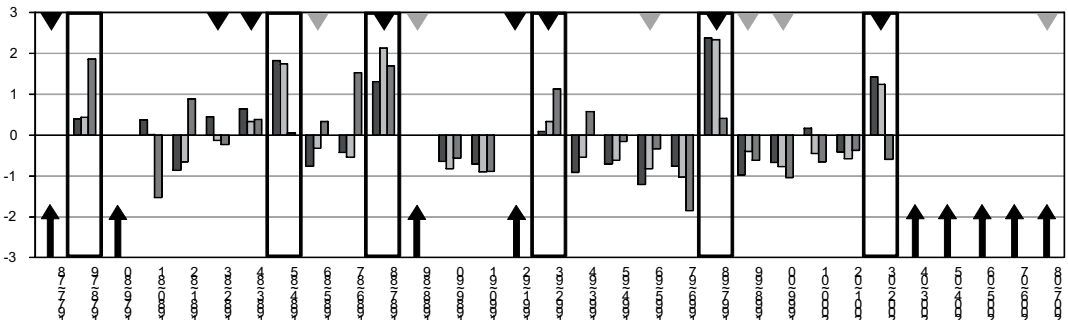

(c)

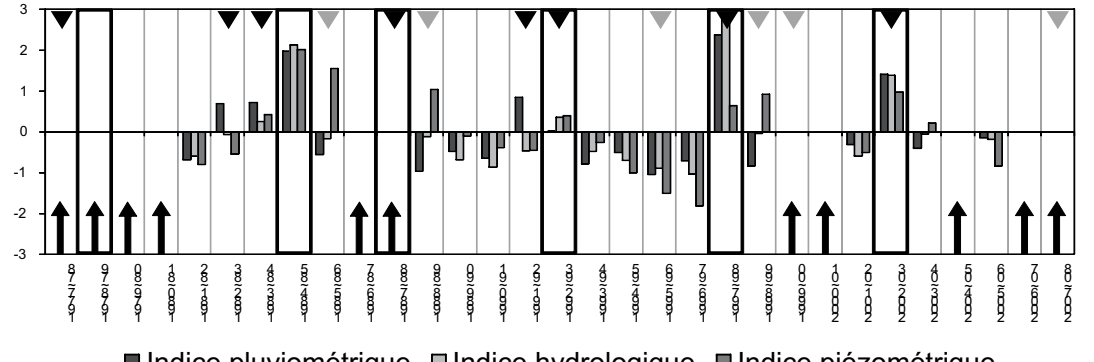

Fig. 2 : Indices pluviométriques, hydrologiques et piézométriques pour (a) le bassin du río Turbio ; (b) le bassin du río Claro ; et (c) le bassin amont de l'Elqui. Les signets noirs et gris en haut indiquent les années hydrologiques associées respectivement à des évènements El Niño et La Niña. Les flèches noires en bas indiquent des années avec lacunes. 


\section{III.2. Analyse des coefficients d'écoulement}

Durant les années humides (IP élevé), les valeurs faibles des CE relevées sur le bassin du río Turbio et le bassin amont de l'Elqui $(<100 \%)$ indiquent un plus faible soutien du compartiment souterrain aux écoulements de surface que durant des années sèches (IP faible). Cela indique également que la part relative des précipitations participant à la recharge du compartiment souterrain augmente au détriment du ruissellement, ce que confirment les variations piézométriques de près de $50 \mathrm{~m}$ sur le piézomètre de référence Bocalume (Fig. 4a). Différentes hypothèses concernant la localisation de cette recharge peuvent être émises : (i) recharge dans le lit majeur du río, au delà d'un seuil de débit correspondant au débordement depuis le lit mineur vers le lit majeur ; (ii) recharge directe par les compartiments souterrains (aquifères) des affluents du río Turbio ; (iii) recharge retardée en pied de versant en provenance de l'horizon altéritique des granites.

Durant les années sèches (IP faible), les importantes valeurs des $C E$ ( $>200 \%$ pendant les évènements $L a$ Niña) peuvent être expliquées par une forte contribution des eaux souterraines à l'écoulement de surface. Cependant, l'analyse des $C E$ sur une succession de plusieurs années sèches montre que la contribution du compartiment souterrain à l'écoulement de surface augmente considérablement durant l'année qui suit un évènement à l'origine d'un $I P$ élevé, puis diminue ensuite progressivement. Ainsi, la relation générale entre compartiments superficiels et souterrains peut être considéré comme un marqueur de la variabilité climatique.

La comparaison des coefficients d'écoulement $C E$ avec les valeurs de précipitation annuelle moyennée sur le bassin du rio Turbio (Fig. 3) montre que lorsque les cumuls annuels de précipitation dépassent approximativement $60-70 \mathrm{~mm}$, les $C E$ ont une valeur systématiquement comprise entre 45 et $50 \%$; ceci indique un équilibre relatif entre recharge du compartiment souterrain et ruissellement. Quand la valeur des précipitations diminue et passe en dessous de ce seuil, les $C E$ augmentent de manière exponentielle, ce qui traduit alors le rôle prépondérant du compartiment souterrain dans le soutien des écoulements de surface.

La Figure 3 permet également d'identifier deux catégories d'années hydrologiques : (i) les années hydrologiques 1977-78, 1980-81, 1982-83, 1983-84 et 1991-92 (cercle gris clair) qui correspondent à des années pour lesquelles la part relative des précipitations participant à la recharge du compartiment est prépondérante par rapport à celle participant au débit du cours d'eau ( $C E$ faibles) ; (ii) les années hydrologiques 1990-91, 1995-96 et 1996-97 (flèches) pour

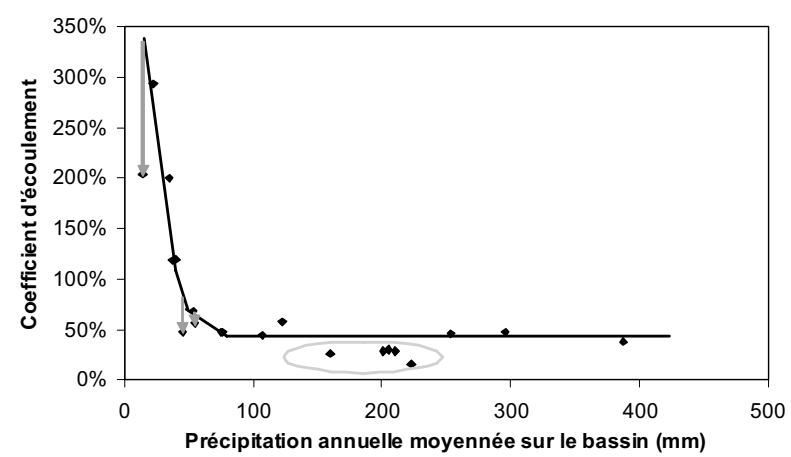

Fig. 3 : Variation des coefficients d'écoulement $d u$ río Turbio en fonction des précipitations annuelles moyennées sur le bassin. lesquelles la contribution du compartiment souterrain à l'écoulement de surface est plus faible que la valeur attendue (courbe), ce qui est probablement la conséquence d'une succession de plusieurs années sèches qui limiterait alors fortement la contribution du compartiment souterrain du fait d'un stress hydrique prononcé (niveau piézométriques très bas). Ainsi, le seuil de $60-70 \mathrm{~mm}$ pourrait être utilisé comme niveau de référence pour la prévention des sécheresses ; lorsque les précipitations sont inférieures à ce seuil, le compartiment souterrain, qui ne bénéficie alors plus d'aucune recharge, soutient les écoulements de surface de façon conséquente, ce qui a pour conséquence d'accroître encore le déficit hydrique.

Si l'on s'intéresse à la variabilité spatiale des $C E$, on constate que les valeurs sont plus élevées sur le bassin du río Claro que sur le bassin du rio Turbio et le bassin amont de l'Elqui ; ceci souligne une contribution plus importante du compartiment souterrain aux écoulements de surface dans le bassin du río Claro, ce que confirment des travaux récents en modélisation hydrologique sur le bassin [Ruelland, 2011]. Comme vu précédemment, l'hypothèse la plus probable est vraisemblablement liée à la géologie, qui permet la recharge du compartiment souterrain (aquifère alluvial) en pied de versant [Dickinson et al., 2004] depuis l'horizon altéritique des granites.

\section{III.3. Dynamique des interactions entre compartiments superficiels et souterrains}

Les mécanismes contrôlant les processus d'écoulement et de recharge ainsi que les dynamiques d'interaction entre compartiments superficiels et souterrains sont maintenant étudiés aux échelles de temps mensuelles et journalières.

Pour les années hydrologiques caractérisées par des $I P$ élevés, trois dynamiques d'interactions entre compartiments superficiels et souterrains ont pu être identifiées à partir des données mensuelles (Fig. 4). La dynamique 1 correspond à une augmentation du niveau piézométrique antérieure à celle des débits. Cette dynamique est observée pour deux événements El Niño (1987-88 et 1997-98) avec des volumes précipités importants sur deux à trois mois. La dynamique 2 correspond à une augmentation du niveau piézométrique qui a lieu après celle des débits. Cette dynamique est observée pour deux événements El Niño (1992-93 et 2002-03), avec des volumes précipités importants répartis sur une longue période (4-6 mois). La dynamique 3 correspond à une augmentation simultanée du niveau piézométrique et des débits, traduisant une même dynamique de transfert entre compartiments superficiels et souterrains. Cette dynamique est observée pour deux années neutres (1978-79 et 1984-85), avec des volumes précipités importants, sur une période de temps très courte (une semaine). Si la tendance climatique actuelle marquée par une plus forte occurrence d'évènements El Niño [IPCC, 2007] se poursuit, les dynamiques 1 et 2 (Figures 4a et $4 \mathrm{~b}$ ) pourraient être plus fréquentes dans le futur.

\section{III.4. Relation entre température et dynamique des flux souterrains et superficiels}

La figure 5 représente les intervalles de température (minimale et moyenne) d'altitude, qui correspondent au déclenchement de la principale augmentation des débits et des niveaux piézométriques. Ces intervalles sont définis par les températures mensuelles antérieures et postérieures du déclenchement de l'augmentation du débit et du niveau piézométrique. 

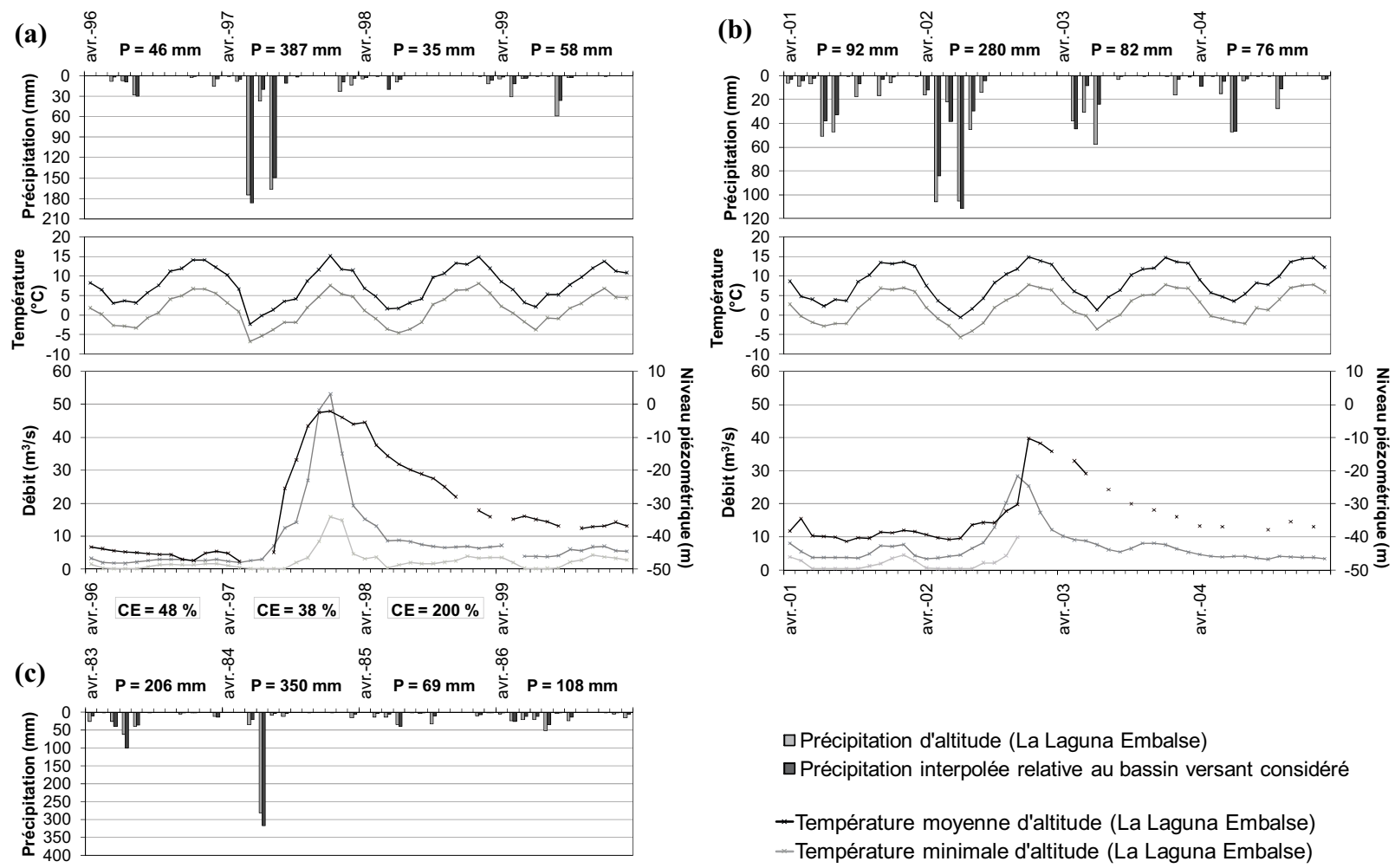

$\square$ Précipitation d'altitude (La Laguna Embalse)

$\square$ Précipitation interpolée relative au bassin versant considéré

- Température moyenne d'altitude (La Laguna Embalse)

- Température minimale d'altitude (La Laguna Embalse)

Fig. 4 : Variations hydro-climatiques mensuelles pour (a) la dynamique 1, (b) la dynamique 2 et (c) la dynamique 3.
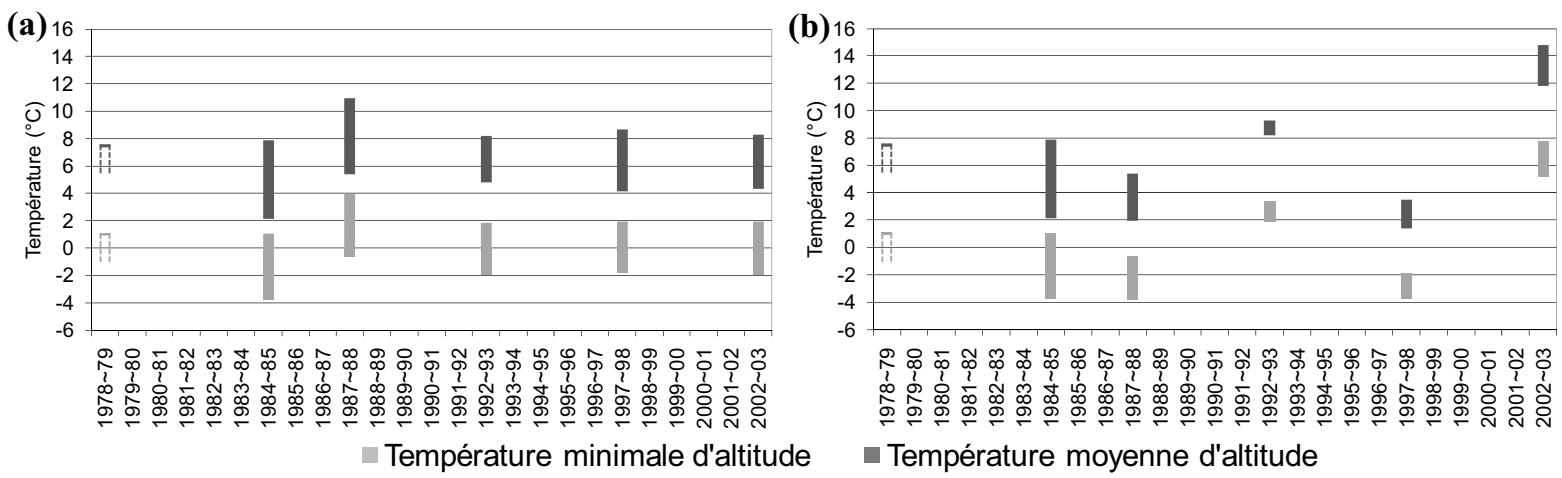

Fig. 5 : Relation entre température et dynamique des flux souterrains et superficiels. Intervalles de température correspondant au début des principales augmentations (a) de débit et (b) de niveau piézométrique. Ces intervalles sont donnés pour les températures minimales et moyennes d'altitude à la station La Laguna $(3160 \mathrm{~m})$.

Les intervalles de température au niveau desquels une augmentation des débits est observée sont relativement stables (Fig. 5a). A l'inverse, l'élévation des niveaux piézométriques semble associée à des intervalles de température beaucoup plus variables (Fig. 5b), avec la dynamique 2 associée à une température notablement plus élevée. La recharge de l'aquifère implique donc des processus dépendant de facteurs autres que la température.

L'augmentation logarithmique des niveaux piézométriques en fonction du temps (dynamique 1, Fig. 4a) traduit une recharge rapide du compartiment souterrain, qui diminue par la suite. Cette dynamique pourrait être la conséquence d'une augmentation douce de la température (Fig. 4a), ce qui entraînerait une fonte plus lente du manteau neigeux et favoriserait dans un premier temps l'infiltration de l'eau de fonte au détriment de son ruissellement.
A l'inverse, l'augmentation exponentielle du niveau piézométrique en fonction du temps (dynamiques 2 et 3 au niveau des Fig. $4 \mathrm{~b}$ et $4 \mathrm{c}$ ) traduit une recharge différée du compartiment souterrain. Pour la dynamique 2, l'augmentation tardive du niveau piézométrique par rapport à celle du débit pourrait être liée à la recharge de l'aquifère provoquée par le débordement du río sur son lit majeur. Par ailleurs, les précipitations étalées sur 4-6 mois génèrent des changements de la structure du manteau neigeux (durcissement et gel), qui peuvent le stabiliser et retarder la fonte de la neige. La figure 6 montre que cette dynamique est dominée par des processus d'écoulement de surface liés à une fonte rapide de la neige, qui se traduisent par une recharge limitée du compartiment souterrain. Une analyse des tendances de température sur la zone d'étude [Souvignet, 2010] montre que les températures 
mensuelles aussi bien maximales que moyennes tendent à l'augmentation. De plus, une tendance de température différente en magnitude entre les hautes et les moyennes altitudes indique une variation possible du gradient thermique, un élément important dans la formation de la neige et des processus de dégel. Si la tendance actuelle d'augmentation de la température se poursuit, l'occurrence de la dynamique 2, moins efficace que les autres dynamiques vis-à-vis de la recharge de l'aquifère, pourrait ainsi augmenter dans le futur.

A échelle de temps journalière, une forte corrélation entre la température et le débit du cours d'eau peut être observée pour les trois dynamiques (Fig. 6). Comme à l'échelle de temps mensuelle, le débit augmente en deux phases : une première augmentation qui peut être attribuée aux premières fontes et une deuxième, correspondant à la principale augmentation, liée à un apport plus conséquent de la fonte des neiges lorsque la température minimale d'altitude dépasse un seuil de $0^{\circ} \mathrm{C}$.

Pour la dynamique 1 (Fig. 6a), la principale augmentation du niveau piézométrique (précédant celle du débit) a lieu quand la température minimale d'altitude se maintient au dessus de $-3^{\circ} \mathrm{C}$ et en dessous de $0^{\circ} \mathrm{C}$ (seuil mentionné ci-dessus) sur une période suffisamment longue (estimée à 1 mois environ). Dans cet intervalle de température, la neige fond et tend à s'infiltrer dans le sous-sol plutôt qu'à ruisseler en surface. Le débit reste alors stable alors que le niveau de la nappe remonte.
Pour la dynamique 2 (Fig. 6b), l'augmentation principale du débit, qui précède l'élévation du niveau piézométrique, a lieu lorsque la température minimale d'altitude dépasse un seuil de $-3^{\circ} \mathrm{C}$ et ne se maintient pas suffisamment longtemps en dessous du seuil de $0^{\circ} \mathrm{C}$. De ce fait, la neige fond plus rapidement et le ruissellement en surface de l'eau issue de la fonte est alors favorisé par rapport à l'infiltration, déclenchant ainsi une forte augmentation du débit. L'augmentation plus tardive du niveau piézométrique pourrait être liée à la recharge de l'aquifère par le débordement du rio Turbio de son lit mineur vers son lit majeur, lorsqu'un seuil de débit de $30 \mathrm{~m}^{3} / \mathrm{s}$ est atteint (Fig. 6b). Une autre raison, comme proposé dans l'analyse mensuelle, serait le changement de la structure du manteau neigeux, rendu plus résistant à la fonte, et retardant ainsi la contribution de l'eau de fonte aux écoulements de surface.

Pour la dynamique 3 (Fig. 6c), l'augmentation concomitante du niveau piézométrique et du débit a lieu lorsque la température minimale d'altitude dépasse $-3^{\circ} \mathrm{C}$ et se maintient dans l'intervalle précédemment identifié (entre $-3^{\circ} \mathrm{C}$ et $0^{\circ} \mathrm{C}$ ), mais pendant une période plus courte que pour la dynamique 1 . Après avoir dépassé le seuil de $-3^{\circ} \mathrm{C}$ pendant plusieurs jours, la température diminue, ce qui provoque un regel de l'eau de fonte. La température augmente ensuite rapidement et dépasse le seuil de $0^{\circ} \mathrm{C}$, déclenchant l'augmentation du débit puis la remontée du niveau piézométrique lorsque le seuil de débit de $30 \mathrm{~m}^{3} / \mathrm{s}$, correspondant au débordement sur le lit majeur, est atteint.

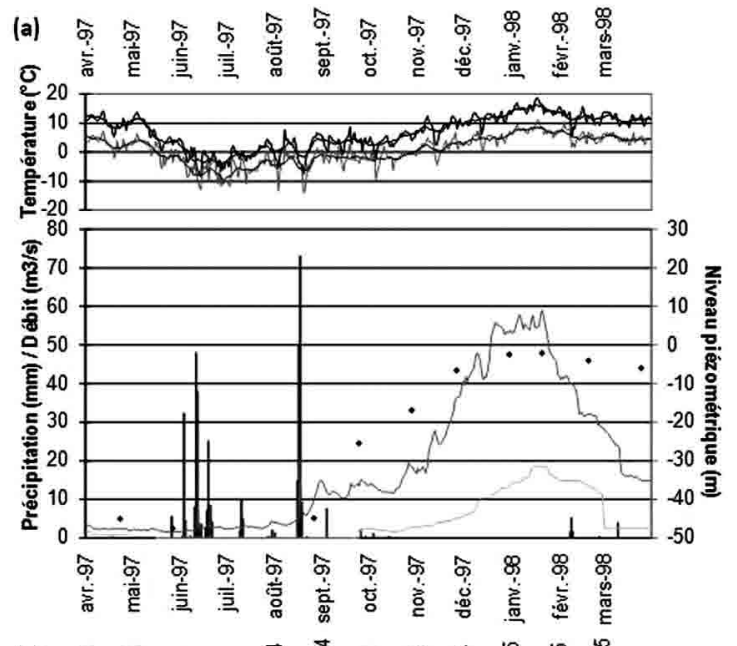

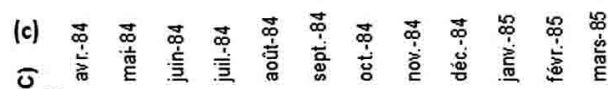

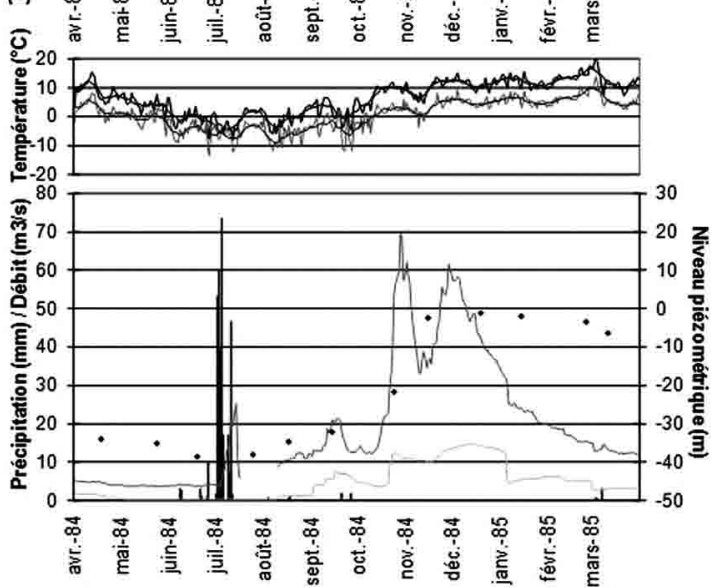

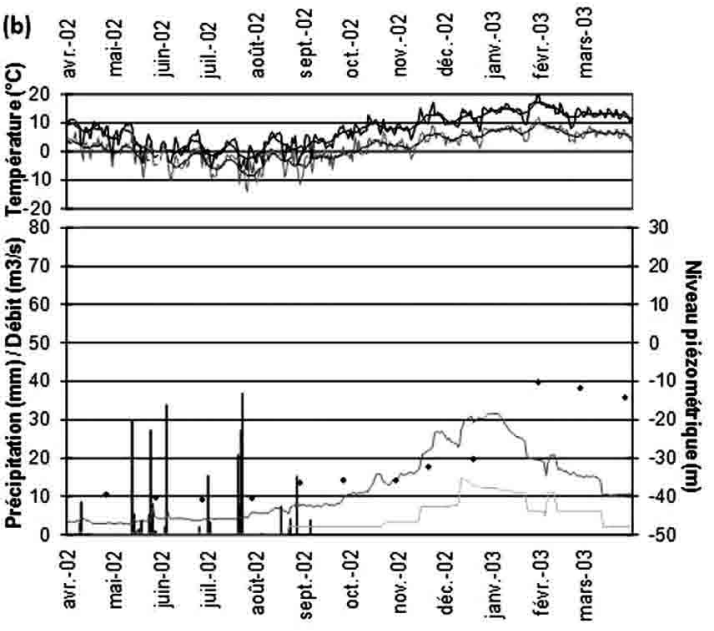

Précipitation interpolée relative au bassin versant considéré -Débit observé

Débit en sortie du barrage de La Laguna

- Niveau piézométrique observé

- Température moyenne d'altitude

- Moyenne mobile décadaire des températures moyennes d'altitude

- Température minimale d'altitude

- Moyenne mobile décadaire des températures minimales d'altitude

Fig. 6 : Variations hydro-climatiques journalières pour (a) la dynamique 1, (b) la dynamique 2 et (c) la dynamique 3. 


\section{CONCLUSION}

L'analyse des coefficients d'écoulement sur les dernières décennies montre que lorsque les cumuls annuels de précipitation sont inférieurs à approximativement $60-70 \mathrm{~mm}$, le relatif équilibre entre écoulements (de surface) et processus de recharge est rompu, ce qui peut générer un déficit hydrologique important. En conséquence, ce seuil peut être utilisé comme un niveau de référence pour la prévention des sécheresses. La variabilité spatiale de ces coefficients d'écoulement montre une contribution plus importante du compartiment souterrain aux écoulements de surface dans un secteur où les granites sont les roches les plus représentées, mettant ainsi en évidence la recharge en pied de versant depuis l'horizon altéritique des granites de l'aquifère alluvial.. Ainsi, de tels secteurs conviendraient à l'exploitation durable des ressources en eau souterraine.

Les analyses à échelle de temps mensuelle réalisées sur le bassin du río Turbio sur la période 1977-2008 permettent d'identifier trois dynamiques différentes d'interactions entre compartiments superficiels et souterrains. Chacune de ces dynamiques est associée à une importante recharge de l'aquifère alluvial suivie par une récession relativement longue des niveaux piézométriques ( 1 à 2 ans) et une plus courte récession des débits (4 à 6 mois). La tendance climatique semble caractérisée par une plus forte occurrence des évènements $E l$ Niño durant les dernières décennies [IPCC, 2007], ce qui pourrait favoriser les dynamiques 1 et 2 , associées à de tels évènements. Cependant, la tendance des températures à l'échelle du bassin favorisererait la moins efficace de ces dynamiques (dynamique 2) en termes de recharge du compartiment souterrain.

Enfin, une analyse journalière a permis d'identifier des seuils de température et des intervalles qui peuvent expliquer le déclenchement de l'écoulement de surface et de la recharge de l'aquifère. Ils pourront servir d'indicateurs pour anticiper les modifications de la ressource en eau superficielle et souterraine, et également être utilisés pour de futures modélisations hydrogéologiques sur le secteur étudié.

\section{REMERCIEMENTS}

Ce travail a été réalisé dans le cadre du projet Ecos-Conicyt C10S01, en partenariat entre HSM et le CEAZA (Centro de Estudio Avanzado en Zonas Aridas, Chile). Nous remercions également la DGA pour la mise à disposition des données hydroclimatiques ainsi que le Conseil Général de l'Hérault pour sa participation au financement des missions de terrain.

\section{REFERENCES}

Cabezas R., Cepeda J., \& Bodini A. (2007) - Descripción cartográfica de la hoya hidrográfica del río Elqui (Región de Coquimbo Chile). Memoria de título para optar al titulo de Ingenior Civil. Universidad de La Serena. 128p

Dickinson J.E., Hanson R.T., Ferre T.P.A., Leake S.A. (2004) Inferring time-varying recharge from inverse analysis of long-term water levels. Water Resources Research. 40 W07403

Favier V., Falvey M., Rabatel A., Praderio E., \& Lopez D. (2009) - Interpreting discrepancies between discharge and precipitation in high-altitude area of Chile's Norte Chico region (26-32 degrees S). Water Resource Research. 45 W02424

Ipcc (2007) - Climate change 2007 : The Physical Science Basis. Contribution of Working Group I to the Fourth Assessment Report of the Intergovernmental Panel on Climate Change (Ed. Solomon S., Qin D., Manning M., Chen Z., Marquis M., Averyt K. B., Tignor M., \& Miller H. L.). Cambridge University Press, Cambridge, United Kingdom and New York, NY, USA. 996 p

Jourde H., Roesch A., Guinot V., \& Bailly-Comte V. (2007) Dynamics and contribution of karst groundwater to surface flow during Mediterranean flood. Environmental Geology Journal. 51(5) 725-730

Luengo P., Oyarzún R., Oyarzún J., Alvarez P., \& Canut De Bon C. (2006) - Aguas subterráneas en macizos rocosos fracturados : su utilización en zonas rurales montañosas del Centro Norte de Chile. VIII Congreso Latinoamericano de Hidrología Subterránea, Asunción, Paraguay

Miranda E., \& Llanca J. C. (2003) - Estudio de las aguas subterráneas en la cuenca del Río Elqui. Memoria de título para optar al titulo de Ingenior Civil. Universidad de La Serena. 203 p

Morales L., Canessa F., Mattar C. \& Orrego R. (2007) Comparison of interpolation methods for detection of microclimatic areas. $5^{\text {th }}$ Int. Symp. Spatial Data Quality (ITC, Enschede, The Netherlands, 12-15 June 2007)

Ruelland D., Brisset, N, Jourde H. \& Oyarzun R. (2011) Modelling the long-term impact of climatic variability on the groundwater and surface flows from a mountainous catchment in the Chilean Andes. In : Cold regions hydrology in a changing climate (Proc. 25 $5^{\text {th }}$ IUGG General Assembly, Melbourne, Australia, 28 June-7 July 2011). IAHS Publ. 346 171-179

Souvignet M., Heinrich J., \& Gaese H. (2010) — Recent Hydroclimatic Trends in the Arid Northern-Central Chile. Global Change : Facing Risks and Threats to Water Resources, (Proc. of the Sixth World FRIEND Conference, Fez, Morocco, October 2010). IAHS Publ. 340 688-694

United States Department Of The Interior (Usdi) - Bureau Of Reclamation (1955) - Elqui Valley Chile, Ground Water Investigation. $92 \mathrm{p}$ 